\title{
The Hamburg/ESO R-process Enhanced Star survey (HERES): Abundances
}

\author{
P. S. Barklem ${ }^{1}$, N. Christlieb ${ }^{2}$, T. C. Beers ${ }^{3}$, V. Hill ${ }^{4}$, \\ J. Holmberg ${ }^{5,6,7}$, B. Marsteller ${ }^{3}$, S. Rossi ${ }^{8}$, F.-J. Zickgraf ${ }^{2}$, \\ and M. S. Bessell ${ }^{9}$ \\ ${ }^{1}$ Department of Astronomy and Space Physics, Uppsala University, Box 515, S 751-20 \\ Uppsala, Sweden \\ email: barklem@astro.uu.se
}

${ }^{2}$ Hamburger Sternwarte, Universität Hamburg, Gojenbergsweg 112, 21029 Hamburg, Germany

${ }^{3}$ Department of Physics and Astronomy and JINA: Joint Institute for Nuclear Astrophysics,

Michigan State University, East Lansing, MI 48824 USA

${ }^{4}$ GEPI, Observatoire de Paris Meudon, F-92125 Meudon Cedex, France

${ }^{5}$ Astronomical Observatory, NBIfAFG, Juliane Meries Vej 30, 2100 Copenhagen, Denmark

${ }^{6}$ Nordic Optical Telescope Scientific Association, Apartado 474, ES-38 700 Santa Cruz de La Palma, Spain

${ }^{7}$ Tuorla Observatory, Väisäläntie 20, FI-21500 Piikkiö, Finland

${ }^{8}$ Departamento de Astronomia, IAG, Universidade de São Paulo, Rua do Matão 1226, 05508-900 São Paulo - SP, Brazil

${ }^{9}$ Research School of Astronomy and Astrophysics, Australian National University, Cotter Rd, Weston, ACT 2611, Australia

\begin{abstract}
We present the results of analysis of "snapshot" spectra (i.e., $\mathrm{R}=20,000$ and $\mathrm{S} / \mathrm{N}=50$ per pixel) of 253 metal-poor halo stars $-3.8<[\mathrm{Fe} / \mathrm{H}]<-1.5$ obtained in the HERES survey. The spectra are analysed using an automated line profile analysis method based on the Spectroscopy Made Easy (SME) codes of Valenti \& Piskunov (1996). Elemental abundances of moderate precision (absolute r.m.s. errors of order $0.25 \mathrm{dex}$ ) have been obtained for 22 elements, C, $\mathrm{Mg}, \mathrm{Al}, \mathrm{Ca}, \mathrm{Sc}, \mathrm{Ti}, \mathrm{V}, \mathrm{Cr}, \mathrm{Mn}, \mathrm{Fe}, \mathrm{Co}, \mathrm{Ni}, \mathrm{Zn}, \mathrm{Sr}, \mathrm{Y}, \mathrm{Zr}, \mathrm{Ba}, \mathrm{La}, \mathrm{Ce}, \mathrm{Nd}, \mathrm{Sm}$, and Eu, where detectable. The results are presented and discussed, particularly trends and scatter in the abundance distributions.
\end{abstract}

Keywords. Stars: abundances - stars: Population II - Galaxy: abundances - Galaxy: evolution Galaxy: halo

\section{Introduction}

In this paper we present and discuss elemental abundances of a large sample of metalpoor halo stars obtained as part of the Hamburg/ESO R-process Enhanced Star (HERES) survey. An overview of the HERES survey has been presented in Christlieb et al. (2004), and in these proceedings. The abundance results presented and discussed here will appear in Barklem et al. (2005). That paper may be consulted for details of the observations and the spectrum analysis, and more detailed discussion of the results.

To briefly summarise, "snapshot" spectra (typically $S / N \sim 54, R \sim 20000, \lambda=3760$ $4980 \AA$ ) of 373 metal-poor stars, here meaning with $[\mathrm{Fe} / \mathrm{H}] \leqslant-1.5$ as judged from medium resolution spectra, have been obtained with VLT2-UVES, with the main goal of finding stars enhanced in the r-process elements through detection of strong Eu II lines. Though the spectra are of what would generally be considered low quality for abundance 

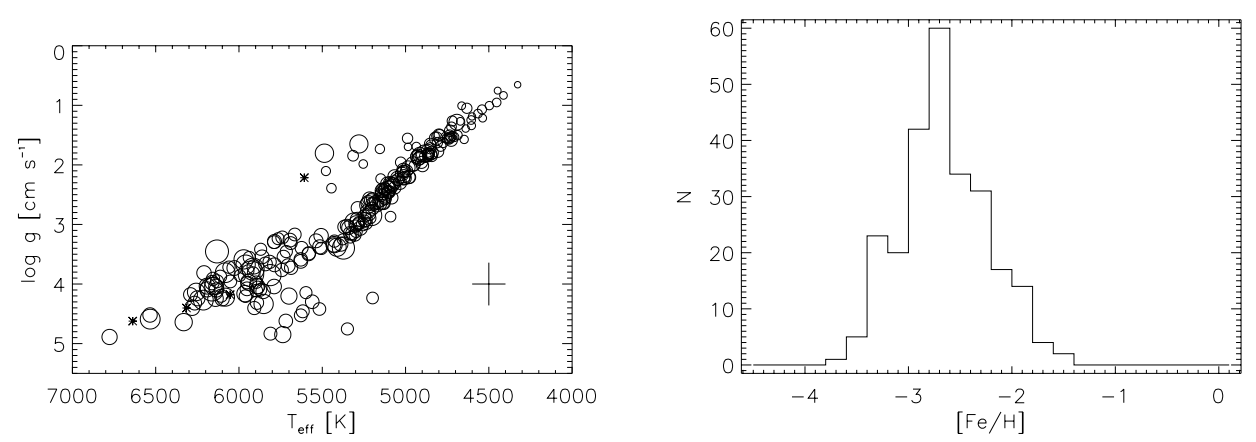

Figure 1. (Left panel) The $T_{\text {eff }}$ versus $\log g$ Kiel diagram for the sample. The diameter of the circle for each star is proportional to $[\mathrm{C} / \mathrm{Fe}]$; cases where $\mathrm{C}$ is undetected are shown with an asterisk. The mean error bar is shown in the bottom right. (Right panel) The distribution of analysed stars in $[\mathrm{Fe} / \mathrm{H}]$.

analysis, they contain a wealth of information and abundances may be derived for a significant number of elements with moderate precision. We have analysed a total of 253 $\dagger$ of the spectra using an automated spectrum analysis technique. The analysed sample of 253 stars covers a large stellar parameter space as shown in Fig. 1.

\section{Spectrum Analysis}

The automated analysis method is described in detail in Barklem et al. (2005). The basic method is based on the Spectroscopy Made Easy (SME) code of Valenti \& Piskunov (1996) which uses a non-linear least squares parameter estimation algorithm to match observed spectra with synthetic spectra in selected comparison wavelength regions. The synthetic spectra are computed under the assumption of LTE using MARCS theoretical 1D plane-parallel model atmospheres (Gustafsson et al. 1975 and subsequent updates).

The new code takes all relevant input data (spectrum, line list, initial guess stellar parameters and abundances) and provides it to the parameter optimisation component of SME without any need for user interaction. The main tasks of the new interface software are, in a completely automated fashion, to extract relevant spectral regions appropriate for the line list, to identify continuum points and normalise the spectra relative to the continuum, to make small adjustments (within the error of the wavelength calibration) to line central wavelengths such that they match the observed line centres, and to reject lines polluted by artifacts such as cosmic ray hits. The stellar effective temperature is determined from photometry, while the remaining model atmosphere parameters $\log g$, $[\mathrm{Fe} / \mathrm{H}]$, and turbulent line broadening parameters are solved using Fe and Ti lines, following which abundances for other elements are solved. For this, a list of spectral features, atomic and molecular data and comparison regions suitable for the stars of interest has been assembled. The list contains several hundred spectral features and includes the latest $f$ values, hyperfine and isotopic structure data from the literature. The results are then extracted, error estimates calculated and valid detections assigned, again by an automated procedure.

$\dagger 72$ stars were rejected because the $\mathrm{CH}$ bands were too strong for the present analysis. 48 stars were too cool or Fe-rich, suspected rotators or binaries, or no photometry was available. 
Table 1. Comparison of measured scatter in Fig. 2 with relative errors in the abundance ratios. For each plot we report the minimum, mean and maximum measured $1 \sigma$ scatter $\sigma_{\text {meas }}$ across the range of $[\mathrm{Fe} / \mathrm{H}]$, which is compared to the average relative error in the abundance ratio $\sigma^{\mathrm{rel}}$. The ratio of the mean measured scatter to the estimated error $\left\langle\sigma_{\text {meas }}\right\rangle / \sigma^{\text {rel }}$ is also reported.

\begin{tabular}{lccccc}
\hline & $\min \left(\sigma_{\text {meas }}\right)$ & $\left\langle\sigma_{\text {meas }}\right\rangle$ & $\max \left(\sigma_{\text {meas }}\right)$ & $\sigma^{\text {rel }}$ & $\left\langle\sigma_{\text {meas }}\right\rangle / \sigma^{\text {rel }}$ \\
\hline$[\mathrm{C} / \mathrm{Fe}]$ & 0.22 & 0.32 & 0.53 & 0.18 & 1.73 \\
{$[\mathrm{Mg} / \mathrm{Fe}]$} & 0.07 & 0.09 & 0.12 & 0.15 & 0.59 \\
{$[\mathrm{Al} / \mathrm{Fe}]$} & 0.17 & 0.20 & 0.21 & 0.17 & 1.13 \\
{$[\mathrm{Ca} / \mathrm{Fe}]$} & 0.08 & 0.09 & 0.10 & 0.15 & 0.59 \\
{$[\mathrm{Sc} / \mathrm{Fe}]$} & 0.09 & 0.11 & 0.13 & 0.16 & 0.69 \\
{$[\mathrm{Ti} / \mathrm{Fe}]$} & 0.08 & 0.09 & 0.12 & 0.15 & 0.61 \\
{$[\mathrm{~V} / \mathrm{Fe}]$} & 0.06 & 0.09 & 0.11 & 0.15 & 0.60 \\
{$[\mathrm{Cr} / \mathrm{Fe}]$} & 0.10 & 0.12 & 0.14 & 0.16 & 0.72 \\
{$[\mathrm{Mn} / \mathrm{Fe}]$} & 0.13 & 0.16 & 0.21 & 0.16 & 1.03 \\
{$[\mathrm{Co} / \mathrm{Fe}]$} & 0.10 & 0.11 & 0.13 & 0.16 & 0.71 \\
{$[\mathrm{Ni} / \mathrm{Fe}]$} & 0.12 & 0.14 & 0.17 & 0.18 & 0.80 \\
{$[\mathrm{Zn} / \mathrm{Fe}]$} & 0.08 & 0.15 & 0.19 & 0.15 & 1.01 \\
{$[\mathrm{Sr} / \mathrm{Fe}]$} & 0.19 & 0.32 & 0.59 & 0.19 & 1.71 \\
{$[\mathrm{Y} / \mathrm{Fe}]$} & 0.21 & 0.25 & 0.32 & 0.17 & 1.47 \\
{$[\mathrm{Zr} / \mathrm{Fe}]$} & 0.13 & 0.19 & 0.23 & 0.16 & 1.18 \\
{$[\mathrm{Ba} / \mathrm{Fe}]$} & 0.37 & 0.50 & 0.69 & 0.18 & 2.77 \\
{$[\mathrm{Eu} / \mathrm{Fe}]$} & 0.21 & 0.37 & 0.62 & 0.16 & 2.34 \\
\hline
\end{tabular}

\section{Results}

It was found that for the typical snapshot spectra, abundances of moderate precision (absolute r.m.s. errors of order 0.25 dex, relative r.m.s. errors of order $0.15 \mathrm{dex}$ ) can be obtained for 22 elements, namely C, Mg, Al, Ca, Sc, Ti, V, Cr, Mn, Fe, Co, Ni, Zn, Sr, Y, $\mathrm{Zr}, \mathrm{Ba}, \mathrm{La}, \mathrm{Ce}, \mathrm{Nd}, \mathrm{Sm}$, and Eu where detectable. Fourteen elements, C, Mg, Al, Ca, Sc, $\mathrm{Ti}, \mathrm{Cr}, \mathrm{Mn}, \mathrm{Fe}, \mathrm{Co}, \mathrm{Ni}, \mathrm{Sr}, \mathrm{Y}$ and $\mathrm{Ba}$, are almost always detectable at the $3 \sigma$ level in the spectra. Another eight elements are analysed, V, Zn, Zr, La, Ce, Nd, Sm and Eu, which can usually only be detected in the spectra of the least metal-poor stars of our sample, spectra with higher than usual $S / N$, if the abundance is enhanced, or a combination of these factors.

It is worth emphasising that some care must be taken in interpreting the results presented here involving elements which are not always, or even usually, detected in our spectra, which may lead to selection effects. Thus, particular care must be taken in interpreting results involving V, Zn, Zr, La, Ce, Nd, Sm and $\mathrm{Eu}$, as the data are incomplete.

\section{Discussion}

\subsection{Abundance Trends and Scatter with Metallicity}

The abundance ratio trends of $[\mathrm{X} / \mathrm{Fe}]$ with $[\mathrm{Fe} / \mathrm{H}]$ have been plotted in Fig. 2 for elements with a reasonable number of detections. The estimated $1 \sigma$ scatter in the $y$-variable is shown (see Barklem et al. (2005) for details). In Table 1 we compare the mean measured scatter in $[\mathrm{X} / \mathrm{Fe}]$, across the range of $[\mathrm{Fe} / \mathrm{H}]$, with the average error estimates. The minimum and maximum measured scatters are also reported to give an indication of the range of variation with $[\mathrm{Fe} / \mathrm{H}]$. Similar results are obtained using $\mathrm{Mg}$ as the standard reference element instead of Fe (see Barklem et al. (2005)).

For $\mathrm{Mg}, \mathrm{Ca}, \mathrm{Sc}, \mathrm{Ti}, \mathrm{Cr}, \mathrm{Fe}, \mathrm{Co}$, and $\mathrm{Ni}$, the measured scatters are slightly less than the scatters expected from the typical estimated relative error in the abundance ratios, suggesting that our computed errors overestimate the real relative error. Bearing in mind 

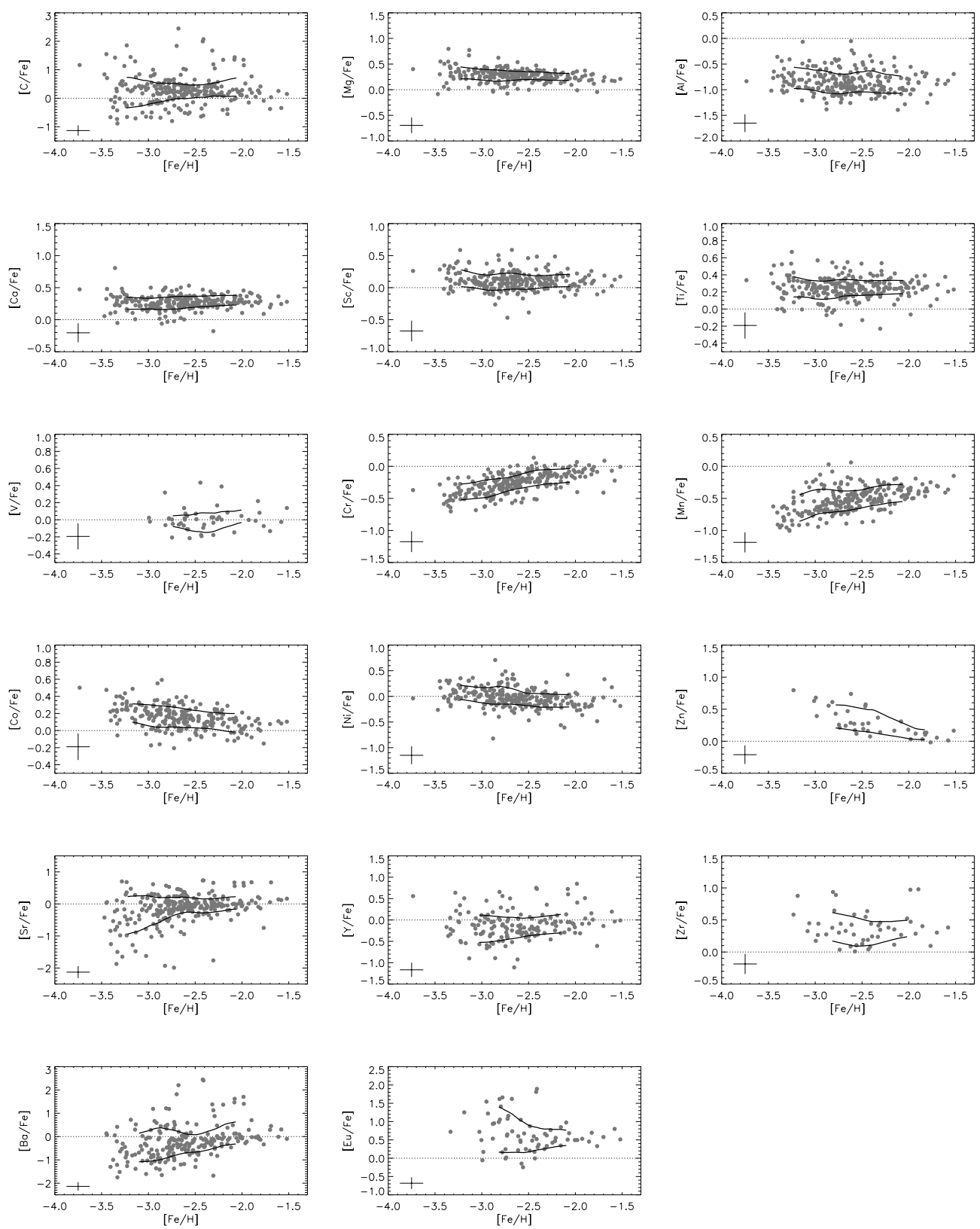

Figure 2. Abundances ratios $[\mathrm{X} / \mathrm{Fe}]$ plotted against $[\mathrm{Fe} / \mathrm{H}]$ for elements with significant numbers of detections. Full lines show estimated $1 \sigma$ scatter. The average relative error bars are shown in the bottom left. Note differing scales on the $y$-axes.

the difficulties in accurately calculating the errors, this suggests that the cosmic scatter is small (or even non-existent) at these metallicities. The results for $\mathrm{Al}, \mathrm{V}, \mathrm{Mn}$ and $\mathrm{Zn}$ are less clear. The elements $\mathrm{C}, \mathrm{Sr}, \mathrm{Y}, \mathrm{Ba}$ and $\mathrm{Eu}$, and perhaps $\mathrm{Zr}$, show scatter at low metallicities, $[\mathrm{Fe} / \mathrm{H}]<-2.5$, significantly larger than can be explained from the errors in the analysis, implying the scatter is cosmic in origin. At higher metallicities the scatter 

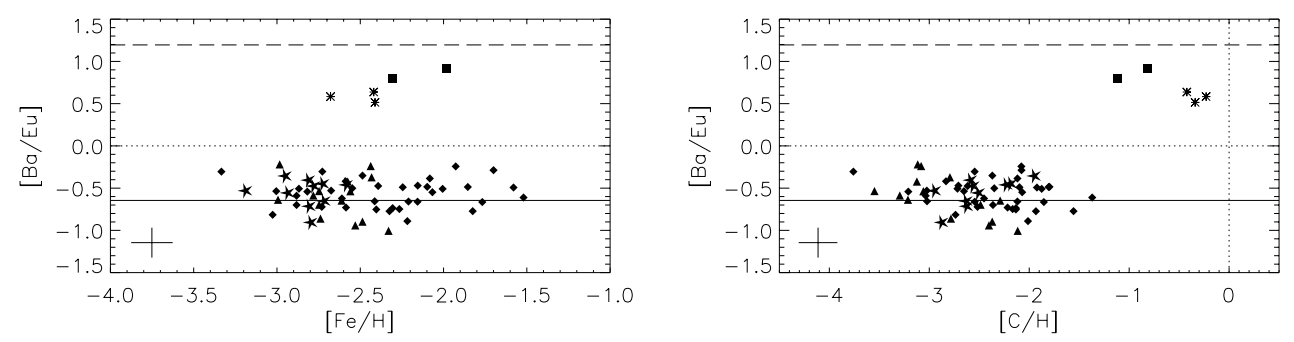

Figure 3. A plot of $[\mathrm{Ba} / \mathrm{Eu}]$ vs $[\mathrm{Fe} / \mathrm{H}]$ (left panel) and $[\mathrm{C} / \mathrm{H}]$ (right panel). The horizontal full line shows the pure solar r-process value, computed from the solar r-process fractions of the Arlandini et al. (1999) stellar model, and the dashed line the solar s-process value. The pure $r-\mathrm{II}$ stars $([\mathrm{Ba} / \mathrm{Eu}]<0,[\mathrm{Eu} / \mathrm{Fe}]>1)$ are shown as stars, the r-I stars $([\mathrm{Ba} / \mathrm{Eu}]<0$, $0.3<[\mathrm{Eu} / \mathrm{Fe}]<1)$ as diamonds, the pure r-process stars without excess r-process elements $([\mathrm{Ba} / \mathrm{Eu}]<0,[\mathrm{Eu} / \mathrm{Fe}]<0.3)$ as triangles, the s-II stars $([\mathrm{Ba} / \mathrm{Eu}]>0,[\mathrm{Eu} / \mathrm{Fe}]>1)$ as asterisks, and the two remaining s-process-rich stars $([\mathrm{Ba} / \mathrm{Eu}]>0,[\mathrm{Eu} / \mathrm{Fe}]<1)$ as squares. The average relative error bar is shown in the bottom left.

among the non-C-rich stars does not greatly exceed that expected from the errors in the analysis, but perhaps indicates some cosmic scatter.

\subsection{Neutron-Capture Elements, Sr-Eu}

Figure 3 plots $[\mathrm{Ba} / \mathrm{Eu}]$ against $\mathrm{Fe}$ and $\mathrm{C}$ abundances. The plots show a clear separation between two groups in the halo, a separation which correlates with $\mathrm{C}$ enrichment. This distinction was first seen in McWilliam (1998), though with fewer stars. The scatter among the pure r-process stars $([\mathrm{Ba} / \mathrm{Eu}]<0)$ is consistent with the observational uncertainties and we thus conclude that the cosmic scatter in $\mathrm{Ba} / \mathrm{Eu}$ among pure r-process halo stars is small. This implies that the $\mathrm{Ba} / \mathrm{Eu}$ abundances produced by the r-process in the early Galaxy are universal. Our result seems to be in disagreement with that found at low metallicities by Truran et al. (2002), who compiled data from a number of studies, and found a large scatter in $\mathrm{Ba} / \mathrm{Eu}$. They noted the difficulties in analysing $\mathrm{Ba}$ in metalpoor spectra due to dependence on hyperfine and isotopic structure and microturbulence. This is particularly problematic when combining data from different studies. Our data on the other hand are homogeneously analysed, but the Eu abundances are incomplete and biased towards stars with strong r-process enhancement.

The production of light neutron-capture elements (particularly Sr, Y, Zr) versus heavy neutron-capture elements (such as $\mathrm{Ba}, \mathrm{Eu}$ ), has become a topic of interest due to evidence of production of the former without significant production of the latter, e.g. McWilliam (1998), Burris et al. (2000), Truran et al. (2002), and others. Figure 4 plots [Sr/Ba] against $\mathrm{Fe}$ and $\mathrm{C}$ abundances. Significant scatter is seen in $\mathrm{Sr} / \mathrm{Ba}$ at low metallicity, as found by McWilliam and Burris et al. Even among the pure r-process stars, a significant amount of scatter is seen at $[\mathrm{Fe} / \mathrm{H}]<-2.5$. The scatter appears to increase quite uniformly with decreasing $[\mathrm{Fe} / \mathrm{H}]$, the data showing quite clear upper and lower boundaries, apart from a small number of outliers which are usually $\mathrm{C}$ enhanced. 

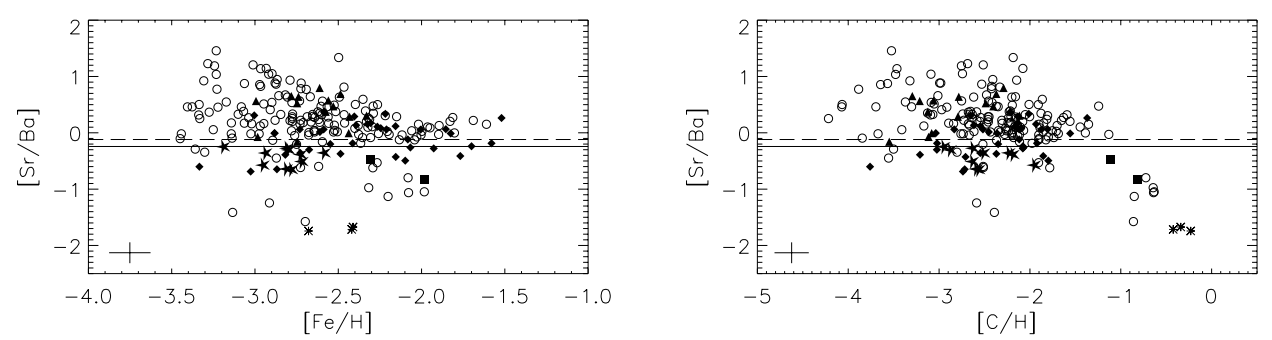

Figure 4. A plot of $[\mathrm{Sr} / \mathrm{Ba}]$ vs $[\mathrm{Fe} / \mathrm{H}]$ (left panel) and $[\mathrm{C} / \mathrm{H}]$ (right panel). Symbols and lines have the same meanings as in Fig. 3, with circles representing stars where Eu is undetected.

\section{References}

Arlandini, C., Käppeler, F., Wisshak, K., et al. 1999, Ap. J. 525, 886

Barklem, P.S., Christlieb, N., Beers, T.C., et al. 2005, A\&SA in press (astro-ph/0505050)

Burris, D.L., Pilachowski, C.A., Armandroff, T.E., et al. 2000, Ap. J. 544, 302

Christlieb, N., Beers, T.C., Barklem, P.S., et al. 2004, A\&A 428, 1027

Gustafsson, B., Bell, R.A., Eriksson, K. et al. 1975, A\&A 42, 407

McWilliam, A. 1998, Ap. J. 115, 1640

Truran, J.W., Cowan, J.J., Pilachowski, C.A., et al. 2002, PASP 114, 1293

Valenti, J.A., Piskunov, N., 1996, A\&SASS 118, 595 